\title{
Il tramonto della pacciada
}

\author{
Mauro Novelli
}

DoI: http://dx.doi.org/10.7359/818-2017-nove

Parole chiave / Keywords: Carlo Emilio Gadda, Carlo Porta, Delio Tessa, Milano, pacciada.

Mi pare opportuno, in apertura, sottolineare la pregnanza del termine milanese impiegato nel titolo di questo intervento. Di norma la pacciada traducibile in italiano con qualche approssimazione come 'scorpacciata' - comporta la presenza di un'allegra compagnia, famigliare o amicale, e si correla perciò tipicamente alle occasioni festive. Ciò, almeno, è quanto si è dato per mezzo millennio e più: durante i secoli in cui ebbe un ruolo cruciale nel plasmare il cliché del milanese 'buseccone', ovvero vorace mangiatore di trippa. Ricostruire la parabola della pacciada consente in effetti di comprendere come anche i più solidi stereotipi identitari possano dissolversi, a contatto con una nuova situazione politica e sociale.

Per un'operazione di questo tipo la letteratura si rivela uno strumento insostituibile, in grado di mettere nitidamente a fuoco l'istruttiva trasformazione subita dal carattere ambrosiano in età contemporanea. Dalla metà del XX secolo intorno allo Stadtgeist milanese si è addensato un paradigma ostile, che ha trovato efficaci rappresentazioni in ambito narrativo. Un romanzo come La vita agra, di Luciano Bianciardi (1962), sciorina un intero campionario di tratti negativi, raccontando una metropoli respingente, abitata da gente avida, guardinga, frettolosa, stacanovista, indifferente, sgarbata. Quest'insieme di presupposizioni, a lungo egemonico e tuttora aleggiante sulla città, si oppone diametralmente al profilo tradizionale dell'ambrosiano affabile, generoso, candido, schietto, incline a darsi buon tempo.

Gli scrittori offrono preziose testimonianze relative alla diffusione e al radicamento di uno stigma che trova il luogo d'elezione nella tavola riccamente imbandita ${ }^{1}$. Una delle attestazioni più precoci è reperibile alla fine

1 Compendio qui la ricostruzione proposta in Novelli 2013, cap. I. Utili spunti in Stella 2005 e Bisi 2011. 
della terza giornata del Decameron, là dove Boccaccio $(1976,258)$ osserva che "ebbevi di quegli che intender vollono alla melanese, che fosse meglio un buon porco che una bella tosa”. L'appetito dei bravi 'busecconi' fu in seguito frequente bersaglio dei poeti satirici: basti rammentare il passo del quinto libro della Secchia rapita - ottava XXXIV, vv. 3-4 - in cui Alessandro Tassoni $(1987,145)$ scherza sull'avanzata nel campo di battaglia della gente di Milano: "ch'ovunque il guardo di lontan volgea / rincarava le trippe, e le frittelle".

Anche in loco questi stereotipi risultano già cristallizzati nel Medioevo e in epoca rinascimentale. Lo provano la fierezza con cui Bonvesin da la Riva $(2009$, 38) nel De magnalibus urbis Mediolani descrive i concittadini, "moribus et vita faceti", e le lodi di Matteo Bandello alla sua città d'adozione:

Milano, come tutti sapete e ogni dì si può vedere, è una di quelle città che in Italia ha pochissime pari in qual si voglia cosa che a rendere nobile, populosa e grassa una città si ricerchi, perciò che dove la natura è mancata, l'industria degli uomini ha supplito, che non lascia che di tutto ciò che a la vita degli uomini è necessario cosa alcuna si desideri, anzi di più v'ha aggiunto la insaziabil natura dei mortali tutte le delicature e morbidezze orientali [...]. Per questo i nostri milanesi ne l'abbondanza e delicatezza dei cibi sono singolarissimi, e splendidissimi in tutti i lor conviti, e par loro di non saper vivere se non viveno e mangiano sempre in compagnia. (Bandello 1952, 116)

Su questi presupposti nasce la maschera di Meneghino, servo arguto, buono, ingenuo, e naturalmente inguaribile ghiottone. Il suo exploit più celebre si incontra a fine Seicento, nel terzo atto, scena terza della commedia dialettale di Carlo Maria Maggi Il barone di Birbanza, quando - costretto a fuggire da Milano - eleva un irresistibile inno alle pietanze, ai vini e alle osterie che avevano allietato le sue giornate (Maggi 1964, 369-372).

Nel XVIII secolo, per indicare la proverbiale ingordigia ambrosiana, si impose una formula - "lupi lombardi" - rintracciabile nelle principali fonti. Vi ricorre ad esempio Carlo Goldoni $(1787,236)$ nelle sue memorie, ricordando una puntata all'osteria della Cazzoeula, così come Giuseppe Baretti, nel XXV capitolo dell'Account of the Manners and Customs of Italy: "The inhabitants of Lombardy, and the Milanese especially [...] resemble likewise the English in their love of good eating, as well in their talking rather too long and too often about it; which has procured them the ludicrous appellation of Lupi Lombardi, that is, Devourers of meat" ${ }^{2}$

2 "Gli abitanti della Lombardia, e i milanesi in particolare [...] assomigliano anche agli Inglesi nel loro amore della buona tavola, così come nell'abitudine di parlarne un po' 
(Baretti 1768, 131-133). Quest'immagine, in genere legata a doppio filo alla bonomia e alla generosità, diventa nei Sepolcri (Foscolo 2010) un capo d'accusa a spregio del "lombardo Sardanapalo", "cui solo è dolce il muggito de' buoi / che dagli antri abdüani e dal Ticino / lo fan d'ozi beato e di vivande" (vv. 58-61). Com'è noto abbondano nell'epistolario foscoliano le invettive contro Milano, "Paneropoli" (città della pànera), o "Butiropoli" (città del burro), e contro i suoi abitanti, il cui spirito si concentra nei loro "stomachi meravigliosi", irrisi in una lettera del 22 gennaio 1815 alla contessa d'Albany $(1956,343)$.

In realtà molte cose erano cambiate in epoca napoleonica, sebbene suoni sin troppo recisa la sentenza di Stendhal (1826, 190-191): "Quatorze années du despotisme d'un homme de génie ont fait de Milan, grande ville renommée jadis pour sa gourmandise, la capitale intellectuelle d'Italie" ${ }^{3}$. In merito vale la pena di rivolgere lo sguardo sul più grande poeta della tradizione dialettale milanese, ovvero Carlo Porta, che negli anni Dieci dell'Ottocento visse una stagione d'oro. Naturalmente sarebbe ingenuo supporre che l'ethos ambrosiano trovi nei suoi versi un limpido specchio. Ma è significativo il modo in cui Porta riformula il cliché del 'buseccone'. In vari pezzi d'occasione lo fa proprio senza esitazioni, celebrando la joie de vivre meneghina, e collegando strettamente ad essa la propria vena poetica, come succede in un frammento forse riconducibile al Brindes de Meneghin all'ostaria scritto nel 1810 per le nozze di Napoleone con Maria Luisa:

Adess mò che hoo ben pacciaa
e ch'el venter l'è tiraa
in sul meder d'on tambor
gh'hoo mej lenna de descor,
dove salten i ganass
mi ghe troeuvi el mont Parnass.
L'estro el scaldi cont el vin
tant pù mej con pù l'è fin.

troppo e troppo spesso, il che ha procurato loro l'appellativo scherzoso di Lupi Lombardi, ossia Divoratori di carne”. Qui e nelle note successive le traduzioni sono di chi scrive.

3 "Quattordici anni di dispotismo esercitato da un uomo geniale hanno fatto di Milano, grande città un tempo celebre per la sua ghiottoneria, la capitale intellettuale dell'Italia”.

4 Framm. 171, vv. 1-8, in Porta 2000, 790, riportato alla grafia standard portiana ("Adesso poi che ho ben mangiato e che il ventre è teso come un tamburo mi cresce la lena di parlare, dove le ganasce ballano io trovo il monte Parnaso. / L'estro lo scaldo col vino, tanto meglio quanto più è fine"). 
Proprio nel Brindes in questione, però, Porta oltrepassa i confini del ditirambo, piegandolo a intenti schiettamente politici. Meneghin, portavoce del popolo ambrosiano, si dà alla baldoria in quanto confida nell'avvento di una fase di pace e prosperità, favorite da un'accresciuta autonomia e da oculate riforme in materia di amministrazione, economia e giustizia. Sotto la vernice del comico premono argomenti serissimi, che altrove Porta affronta in chiave di satira, scagliandosi contro il parassitismo ecclesiastico e nobiliare.

Il cibo assume in quest'ottica un rilievo strategico, come reagente in grado di palesare le ipocrisie della società: si può ricordare al riguardo il Meneghin biroeu di ex monegh, dove il servo risponde piccato a un prete obeso che rimprovera la voracità dei poveracci nelle bettole. La Milano portiana gravita intorno al vivace mercato del Verziere, "scora de lengua" ${ }_{5}$ sede del più autentico spirito ambrosiano, contrapposto in On funeral alla chiesa, luogo dello spirito ridotto a mercato dalla mediocrità del clero. È tra i banchi del Verziere che la Ninetta fa i conti con le leggi dell'interesse e del desiderio, in cui si perde, spolpata dal cinico Pepp, che la sfrutta sino all'osso. La ribellione, tardiva e velleitaria, scatta solo quando l'amante ne intacca l'onestà di prostituta, mettendo in giro la voce che sia impestata:

Ma se pò dà on trattà pussee balloss, on'azion pussee infamma e de cortell? Pacciamm tutt, godemm tutt, e de maross taccamm anca in l'onor, toeumm anca quell? ${ }^{6}$

In un altro monologo attribuito a un personaggio popolare, il Lament del Marcbionn di gamb avert, la perfida Tetton usa la convivialità per realizzare il suo inganno: prima seduce il malcapitato ciabattino con un brindisi malizioso, poi gli estorce la promessa matrimoniale tra vini e risotto, a tarda ora. I ricettacoli del gaio e sincero animo ambrosiano si trasformano in trappole sinistre: di lì a poco anche Manzoni, nei Promessi Sposi, avrebbe riempito le osterie di frottole e trabocchetti.

Guai agli sprovveduti: intanto però i tempi in cui "Ai milanesi si negava ogni altra lode che quella di 'lupi lombardi', di 'populo epulante”"

5 On funeral, v. 2, in Porta 2000, 474 (“scuola di lingua”). Sull'importanza del Verziere in Porta cf. Banfi 1970, 193-205.

6 La Ninetta del Verzee, vv. 333-336, in Porta 2000, 140 ("Ma può esistere un comportamento più mascalzone, un'azione più infame e da coltello? Papparmi tutto, godermi tutto e per giunta attaccarmi anche nell'onore, levarmi anche quello?"). 
sono ormai lontani, come registra soddisfatto Carlo Cattaneo $(1999,319)$ nel 1836. L'amplissimo spazio dedicato da Francesco Cherubini a termini, locuzioni e proverbi relativi alla sfera alimentare, nel suo Vocabolario milanese-italiano (1839-1843), tramanda ai posteri le vestigia di un ricco corpus tradizionale avviato al declino. Lo stereotipo del 'buseccone' viene confinato ai gradini più bassi della società ambrosiana. In prossimità del Quarantotto l'ingordigia tende a perdere l'aura di indulgenza che da sempre la circondava. Non è compatibile con l'operosa sobrietà predicata dalle borghesie in ascesa, né con i nuovi ideali nazionali, che inducono a percepire sciali, risate e banchetti come espedienti per addormentare le coscienze, funzionali all'occupazione straniera.

All'indomani dell'Unità, solo nello sguardo di alcuni stranieri sopravvive l'idea di Milano come città 'grassa' e gaudente. Incurante dei cinquant'anni trascorsi dagli entusiasmi di Stendhal, Hippolyte Taine può ancora farne "la patrie de la bonhomie et du plaisir; considérer le travail et les préoccupations graves comme une corvée qu'il faut réduire le plus qu'on peut, s'amuser, rire, faire des parties de campagne, être amoureux, non pas à la manière des soupirans, voilà leur façon de prendre la vie" ${ }^{7}$ (Taine 1866, 506). Mark Twain, poco più tardi, arriva a comporre un elogio della calma percepita fra gli abitanti, così distante dalla frenesia degli americani: "we even carry our business cares to bed with us [...]. / I do envy these Europeans the comfort they take. When the work of the day is done, they forget it" ${ }^{8}$ (Twain 1869, 186-187).

In realtà la metamorfosi del carattere ambrosiano, che ci porta a leggere con un sorriso queste parole, era già iniziata da tempo. Il Risorgimento dà il colpo decisivo, determinando una rotazione della prospettiva, che non tarda a produrre effetti. Milano è convertita da capoluogo di una provincia meridionale dell'impero asburgico a 'capitale morale' della nuova Italia. L'unificazione politica della penisola porta in dominante i valori di laboriosità e concretezza sui quali si costituisce un mito destinato ad arrivare ai nostri giorni, puntualmente accompagnato da discussioni e polemiche (cf. Decleva 1980; Spinazzola 1981; Rosa 2015). I volumi nati in margine all'Esposizione Nazionale del 1881 - in particolare Milano 1881

7 "La patria della bonomia e del piacere; considerare il lavoro e le preoccupazioni serie come una fatica da ridurre il più possibile, divertirsi, ridere, fare scampagnate, innamorarsi senza spasimare, ecco come prendono la vita”.

8 "Noi addirittura ci portiamo a letto le preoccupazioni legate agli affari [...]. Invidio a questi europei la tranquillità che si concedono. Quando il lavoro della giornata è fatto, lo scordano". 
(Ottino), Milano e i suoi dintorni (Civelli) e i quattro tomi di Mediolanum (Vallardi) - testimoniano il rapido assestamento di un sistema ideologico in cui ghiottoneria e giovialità spensierata, connotati primari della Milano d'antan, sono vituperati, rimossi o abbassati al rango di vassalli, in quanto effetti leciti di una faticosa e meritata prosperità. Non stupisce perciò scoprire che i milanesi a quest'altezza cronologica prendono per un'offesa il vecchio nomignolo di 'busecconi', teste il veneziano Raffaello Barbiera, che si diverte nel perseverare:

del resto, voi potete esaltare Milano come meglio vi piace, ma non colpirete mai nel giusto segno se non ricorrete al suo epicureismo. Milano ha la beneficenza ed ha il Duomo; ha la Scala ed ha il Cenacolo da Vinci; ma la sua caratteristica ricchezza è uno stomaco di bronzo invidiabile. (Barbiera 1881, 509)

Proprio questo stomaco consente di accettare le ingiustizie denunciate dai cosiddetti 'palombari sociali' in indignati articoli, libri e pamphlet. Valga a esempio il parodico "addio" manzoniano con cui Paolo Valera apre Gli scamiciati, intonato da un gruppo di disperati che si allontanano dalla darsena in barca, lungo il naviglio: "Addio, città della busecca, dove molti muoiono per mancanza d'alimento e molti d'indigestione, nota a chi è cresciuto nel tuo grembo e ti ha cercato invano un boccone di pane; [...] addio!" (Valera 1881, 4). Anche Giovanni Verga, nelle novelle milanesi riunite nel 1883 in Per le vie, manda in frantumi ogni ipotesi di convivialità serena, ritraendo gli effetti deteriori della modernità urbana. L'ironia amara di un pezzo come L'osteria dei 'buoni amici' - insegna sotto cui si trovano soltanto violenza e abbrutimento - lo prova con didascalico rigore. Tonino, uscito ubriaco da una cena fra amici all'osteria del titolo, quasi ammazza un merciaio in una rissa. Arrestato, deve scontare l'ostilità della famiglia, che gestisce un banco di verdure al Verziere e non vuole rogne. Infine finisce di nuovo in galera, tradito dai suoi "buoni amici" per un miserabile lucro. Ancora più esemplare l'indifferenza in cui nell'Ultima giornata si consuma il dramma di un disoccupato, che offusca per un attimo la baldoria festiva nelle bettole del suburbio:

Come il sole tramontava l'ombra del cadavere si allungava, dai piedi senza scarpe, a guisa di spaventapassere, e gli uccelli volavano via silenziosi. Dalle osterie vicine giungevano allegri il suono delle voci e la canzone del Barbapedana. In fondo al cortile, dietro le pianticelle magre in fila si vedevano saltare e ballare le ragazze scapigliate. E quando il carro che portava i resti del suicida passò sotto le finestre illuminate, queste si oscurarono subito dalla folla dei curiosi che s'affacciavano per vedere. Dentro, l'organetto continuava a suonar il valzer di Madama Angôt. (Verga 2003, 110-111) 
Presto scacciata, la visione del suicida torna ad affacciarsi a tarda sera fra i crocchi dei giovani, provocando un vago turbamento, che non annulla l'aggressività latente, pronta a sciogliersi in litigi sanguinosi. Non a caso in un notevole racconto di poco posteriore, Tentazione!, la fine di una domenica simile, trascorsa fra amici in un'osteria fuoriporta ("c'era lì una gran folla, e quel dell'organetto, e quel della chitarra, e ragazze che strillavano sull'altalena”: Verga 2008, 193), innesca il crescendo che porta allo stupro e all'assassinio di una contadina.

Volta invece innanzitutto al pittoresco è la "fisiologia della capitale morale" ricostruita in Il ventre di Milano (Barilli et al. 1888) da una "società di letterati" capeggiata da Cletto Arrighi. Fra bagliori d'ironia e nostalgie cocenti i vecchi stereotipi possono celebrarvi gli ultimi trionfi, prima di scegliere come definitivo terreno d'elezione la poesia dialettale, dove sono sopravvissuti - via via sempre più ignorati - lungo il XX secolo. In quest'ambito i richiami all'opera di Carlo Porta andarono presto assumendo valenze regressive. Non conta che l'operosità borghese costituisca nei suoi versi un polo positivo, sistematicamente opposto all'indolenza in cui sprofondano il peggior clero e la peggior nobiltà: Porta funzionò da farmaco per chi cercava nella letteratura un rimedio adatto a esorcizzare l'avanzata delle spregiudicatezze capitalistiche nel rombante Milanon, così diverso dal perduto Milanin del primo Ottocento.

Anche Delio Tessa, il maggiore poeta dialettale lombardo del Novecento, nelle prove giovanili non risulta estraneo a queste dinamiche. Ma nei capolavori la lezione del maestro si fa ben più profonda e pervasiva, sebbene sia direttamente evocato solo nel poemetto che gli dedicò negli anni del fascismo trionfante. In apertura Tessa allestisce un contesto rassicurante, immaginando una "gran pacciada" natalizia, coronata da una lettura portiana, affidata come da tradizione al patriarca famigliare:

Contra i melanconij, contra i magon

rezipe, el me zion, rezipe i rimm del Porta; el pà Carloeu

dopo la gran pacciada

per el Santo Natal... (e ravioeu, pollin, torron, mostarda) ... in cà Marianna

ultem piatt de portada - varda, varda

l'è chì, largo, l'è chì! - pas e legria!! ${ }^{9}$

9 A Carlo Porta, vv. 1-12, in Tessa 1988, 391: "Contro le malinconie, contro le tristezze recipe, caro zione, recipe le rime del Porta; il papà Carlo dopo la grande abbuffata 
Parrebbe una commossa rievocazione di quella "civiltà alimentare" ambrosiana cui Alberto Savinio in Ascolto il tuo cuore, città dedicò pagine memorabili, nella convinzione che "la civiltà quando arriva al suo àpice diventa naturalmente conviviale e la tavola centro della vita, anche spirituale" (Savinio 1988, 127). I fantasmi del Bongee, del Marchionn e degli altri personaggi, emersi da una cinematografica nebbia grazie alle parole dello zio, devono però lasciare il posto agli incubi diabolici del Novecento, che sostituisce le damazze con boriose donne di banchieri e industriali, scese da grosse automobili tra i fumi pestilenziali dei gas di scarico. Il farmaco portiano è ormai un inutile placebo. Tessa prima rivolge una dolente apostrofe a "Carlin", inorridito dal ritorno del randello, non più asburgico ma fascista. Poi, certo dell'impossibilità di estirpare un male penetrato troppo a fondo nel tessuto cittadino, innalza un brindisi nient'affatto innocente, riprendendo il modulo portiano per esaltare i valori del regime, con sarcasmo antifrastico:

slarga el coeur, - semm fradeij,
tucc fradeij se Dio voeur!
semm gioven e semm beij,
semm grass come porsceij
semm tucc ona fameija de bagaij,
coeur avert - quant al rest,
lassa perd - che l'è bon
per i caij; - se el fattor
quand l'occor - l'è tant bon
de dà el fen - alla vacca,
coss te ven, - coss te calla
ancamò? coss te voeutt?
Libertaa? Oh che balla!
tocca no che l'è cacca! ${ }^{10}$

Meglio chiudere il libro di Porta, e stare all'erta. Anche i muri hanno orecchi e dappertutto - come si legge in Anno VIII - "l'è on spettacol de roij e de stabieij" ${ }^{11}$. L'utopia di una città-famiglia, solidale e coesa, è pic-

per il Santo Natale... (e ravioli, tacchino, torrone, mostarda)... ultimo piatto di portata in casa Mariani - guarda, guarda, è qui, largo, è qui! - pace e allegria!!”.

10 A Carlo Porta, vv. 167-180, in Tessa 1988, 403 ("allarga il cuore, siamo fratelli, tutti fratelli se Dio vuole! Siamo giovani e belli, siamo grassi come porcelli, siamo tutti una famiglia di ragazzi, cuore aperto - quanto al resto, lascia perdere, va bene per i calli; se il fattore, quando serve, è così buono da dare il fieno alla vacca, cosa cerchi, cosa ti manca ancora? Cosa vuoi? Libertà? Oh che balla! Non toccare, è cacca!”).

11 Anno VIII, v. 11, in Tessa 1988, 423 (“è uno spettacolo di maiali e porcili”). 
conata con veemenza. In un altro componimento coevo, "I cà", un operaio disoccupato urla la sua disperazione - “... Demm de mangià.a.a.!..." 12 _ ma le urla rimbalzano come sassi alle finestre di casermoni squadrati, in uno scenario degno di Sironi. All'interno una famiglia benestante, finita la cena, ascolta tranquillamente un concerto trasmesso dalla radio, che copre i rumori provenienti dalla strada. La nuova borghesia non sente i bisogni dei poveri: anzi, convertita all'italiano del regime, ormai nemmeno li capisce. Per sottrarsi a questa frattura, sociale e linguistica insieme, l'avvocato Tessa resta avvinghiato al dialetto e in De là del mur - poemetto completato intorno al 1930 - inforca la bicicletta per dirigersi fuori città. L'approdo al tradizionale rifugio del letterato è però sconfortante: le campagne, raschiata la pervasiva retorica fascista, si rivelano abitate da bruti storditi dal vino, che santificano la sera del di di festa giocando a carte o alla morra nelle osterie, fra urla e bestemmie rauche.

Spartiacque fondamentale per Tessa era stata la Grande guerra, che lo separò una volta per tutte dalla belle époque, dissolvendo - insieme allo stato liberale - gli ideali di pace e fratellanza assimilati in gioventù sulle pagine dell'ultimo Tolstoj. Non stupisce dunque che abbia dato alla poesia italiana un unicum di valore altissimo come Caporetto 1917: 410 versi che insistono con oltranza espressionistica sul momento più tragico del conflitto, colto dalle retrovie, quando Milano, a una settimana dalla rotta, si trovò a celebrare il giorno dei morti col fiato sospeso, mentre dal fronte si rincorrevano notizie contraddittorie.

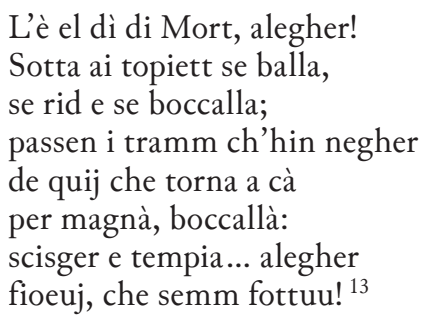

Evocati dal poeta, che rientra dal cimitero insieme a una folla vociante, ceci e tempia - pietanze tradizionali della pacciada del 2 novembre - acquisiscono una sfumatura quanto mai sinistra, mescolati a un'allegria disperata e funebre, nella cornice delle esclamazioni ossimoriche che aprono

12 I cà, v. 20, in Tessa 1988, 305 (“... Datemi da mangiare!...”).

13 Caporetto 1917, vv. 8-15, in Tessa 1988, $55-56$ ("È il giorno dei morti, allegri! Sotto le pergole si balla, si ride e si sbevazza; passano i tram neri di quelli che tornano a casa per mangiare e sbevazzare: ceci e tempia... allegri / ragazzi, che siamo fottuti!"). 
e chiudono il brano. In mezzo i tram carichi di gente, le bevute e i balli sfrenati sotto le pergole. Tutt'intorno, i giovani in procinto di partire per il fronte, che cantano a squarciagola per vincere l'angoscia.

Negli stessi giorni, insieme a migliaia di commilitoni, un ufficiale milanese catturato a Caporetto veniva trasferito in un lager tedesco, dove avrebbe patito oltre al freddo una fame lancinante, come racconta nel Giornale di guerra e di prigionia. Anche Carlo Emilio Gadda visse la Grande guerra come una ferita immedicabile, che straziò tanto gli affetti privati (vi morì il fratello Enrico), quanto gli ideali patriottici che l'avevano guidato nelle manifestazioni a favore dell'intervento. Oltrepassa gli intenti di questo contributo un'analisi puntuale del ruolo conferito al cibo nella pagina gaddiana ${ }^{14}$. Appare invece opportuno concentrare l'attenzione sui due "disegni milanesi" dell'Adalgisa spiccati dalla Cognizione del dolore, per verificare la torsione drammatica cui viene sottoposto il connotato storico della ghiottoneria.

Se nel più portiano dei suoi libri Gadda torna al "clima unto e fraterno", "lieto di ceci e verze" della Milano conosciuta in gioventù, oscillando fra sorriso e nostalgia, in Strane dicerie contristano i Bertoloni il clima si incupisce. "Vorace, e avido di cibo e di vino: crudele: e avarissimo", don Gonzalo Pirobutirro - alter ego dell'autore - si abbandona a crapule mostruose in un lercio "stambugio", dove si narra abbia rischiato la morte pur di trangugiare

un riccio, altri sostenevano un granchio, una specie di scorpione marino ma di colore, anziché nero, scarlatto, e con quattro baffi, scarlatti pure essi, e lunghissimi, come quattro spilloni da signora, due per parte, oltre alle mandibole, in forma di zanche, e assai pericolose loro pure; qualcuno favoleggiava addirittura di un pesce-spada o pesce-spilla; eh, già! piccolo, appena nato; ch'egli avrebbe deglutito intero (bollitolo appena quanto quanto, ma altri dicevano crudo), dalla parte della testa, ossia della spada: o spilla. Che la coda poi gli scodinzolò a lungo fuor dalla bocca, come una seconda lingua che non riuscisse più a ritirare, che quasi quasi lo soffocava. (Gadda 1987, 85-86)

Il narratore elenca puntigliosamente ogni vivanda divorata dall'iracondo e misantropo bidalgo, nel corso di un rito solitario che nulla ha da spartire con gli ilari conviti della tradizione ambrosiana. Ed è ancora in solitudine, dinanzi a una scodella di zuppa e a "tre peperoncini verdastri, vizzi" serviti dalla madre su un piatto slabbrato, nella penombra silenziosa, che si condensa nella sua mente la visione di un'animata pacciada, in Navi

14 Sul tema cf. almeno Biasin 1991. 
approdano al Parapagàl. La scena non si svolge in un'osteria ma in un ristorante di signori, felici di ostentare la loro ricchezza, impettiti, compiaciuti, galvanizzati dalla deferenza dei camerieri. Nelle mani dell'ingegner Gadda - "polpetta saltata via dalla padella lombarda" ${ }^{15}$, a Firenze, poi a Roma - i 'busecconi' si trasformano alla fiamma del sarcasmo in "sibariti in trentaduesimo", in "manichini ossibuchivori" (Gadda 1987, 346-347): tronfi campioni di quella mediocre borghesia in cui si spegne il mito della capitale morale.

Quanto all'oggi, a dar retta alla solerzia della Rete, la pacciada sopravvive soltanto come titolo di un libro di Gianni Brera e Luigi Veronelli (1973), uscito da quarant'anni e più. A sfogliarlo, peraltro, ci si accorge presto di come Brera alla dimensione urbana preferisca l'elegia campagnola, pesantemente condita di lodi alla calunniata ma formidabile 'etnia' lombarda. L'antico blasone della ghiottoneria ambrosiana è andato sbiadendo sino a risultare irriconoscibile. Da questo punto di vista l'appuntamento dell'Expo, in cui Milano si propone di spiegare come "nutrire il pianeta”, finisce col somigliare a un ritorno del rimosso. Nel frattempo le pacciade di cui si parla più spesso sono quelle - metaforiche o meno - di politici e imprenditori corrotti. E qui, davvero, c'è poco di che stare allegri.

\section{RIFERIMENTI BIBLIOGRAFICI}

Bandello, Matteo. (1934) 1952. "Le novelle". In Tutte le opere, a cura di Francesco Flora, vol. I. Milano: Mondadori.

Banfi, Antonio. 1970. "Rileggendo il Porta. Un grande poeta italiano nella Milano dell'Ottocento". In Scritti letterari, a cura di Carlo Cordiè, 193-205. Roma: Editori Riuniti.

Barbiera, Raffaello. 1881. "Milano epicurea”. In Milano 1881, 505-519. Milano: Ottino.

Baretti, Giuseppe. 1768. An Account of the Manners and Customs of Italy; with Observations on the Mistakes of Some Travellers, with Regard to the Country, vol. 2. London: T. Davies, L. Davis, C. Reymers.

Barilli, Aldo, Ferdinando Fontana, Leo Speri, Otto Cima, et al. 1888. Il ventre di Milano. Fisiologia della capitale morale. Milano: Aliprandi, 2 voll.

Bianciardi, Luciano. 1962. La vita agra. Milano: Rizzoli.

15 Si tratta di un'autodefinizione, testimoniata da Cattaneo 1973, 45. 
Biasin, Gian Paolo. 1991. "La cornucopia del mondo". In Gian Paolo Biasin, I sapori della modernità. Cibo e romanzo, 113-139. Bologna: il Mulino.

Bisi, Lucia. 2011. Nutrire Milano. Storia e paesaggio dell'alimentazione in città. Milano: Skira.

Boccaccio, Giovanni. 1976. Decameron, edizione critica a cura di Vittore Branca. Firenze: Accademia della Crusca.

Bonvesin da la Riva. 2009. Le meraviglie di Milano, a cura di Paolo Chiesa. Milano: Fondazione Valla - Mondadori.

Brera, Gianni, e Luigi Veronelli. 1973. La pacciada. Mangiarebere in pianura padana. Milano: Mondadori.

Cattaneo, Carlo. 1999. "Sui milanesi e il loro dialetto". In Scritti su Milano e la Lombardia, a cura di Ettore Mazzali, 317-328. Milano: Rizzoli.

Cattaneo, Giulio. 1973. Il gran lombardo. Milano: Garzanti.

Cherubini, Francesco. 1839-1843. Vocabolario milanese-italiano. Milano: Imperial Regia Stamperia, 4 voll.

Decleva, Enrico. 1980. "L'esposizione del 1881 e le origini del mito di Milano”. In Dallo Stato di Milano alla Lombardia contemporanea, vol. I, a cura di Silvia Pizzetti, 181-211. Milano: Cisalpino-Goliardica.

Foscolo, Ugo. 1956. Epistolario, a cura di Plinio Carli, vol. V. Firenze: Le Monnier.

Foscolo, Ugo. 2010. Dei Sepolcri. Carme, edizione critica a cura di Giovanni Biancardi e Alberto Cadioli. Milano: Il Muro di Tessa.

Gadda, Carlo Emilio. 1987. La cognizione del dolore, edizione critica a cura di Emilio Manzotti. Torino: Einaudi.

Goldoni, Carlo. 1787. Mémoires pour servir à l’histoire de sa vie et à celle de son théâtre. Paris: Duchesne.

Maggi, Carlo Maria. 1964. Il teatro milanese, a cura di Dante Isella. Torino: Einaudi.

Novelli, Mauro. 2013. Divora il tuo cuore, Milano. Carlo Porta e l'eredità ambrosiana. Milano: il Saggiatore.

Porta, Carlo. (1975) 2000. Poesie, a cura di Dante Isella. Milano: Mondadori.

Rosa, Giovanna. 2015. Il mito della capitale morale. Identità, speranze e contraddizioni della Milano moderna. Milano: Rizzoli.

Savinio, Alberto. (1944) 1988. Ascolto il tuo cuore, città. Milano: Bompiani.

Spinazzola, Vittorio. 1981. “La 'capitale morale’. Cultura milanese e mitologia urbana”. Belfagor XXXVI (3): 317-327.

Stella, Angelo. 2005. “'Povere cene' di Lombardia”. In Gian Luigi Beccaria, Angelo Stella, e Ugo Vignuzzi, La linguistica in cucina, 25-101. Milano: Unicopli.

Stendhal. 1826. Rome Naples et Florence, vol. I. Paris: Delaunay.

Taine, Hippolyte. 1866. Voyage en Italie, vol. II. Paris: Hachette.

Tassoni, Alessandro. 1987. La secchia rapita, edizione critica a cura di Ottavio Besomi, vol. 1: Prima redazione. Padova: Antenore. 
Tessa, Delio. (1985) 1988. L'è el dì di Mort, alegher! De là del mur e altre liriche, a cura di Dante Isella. Torino: Einaudi.

Twain, Mark. 1869. The Innocents Abroad or The New Pilgrims' Progress. Hartford: American Publishing Company.

Valera, Paolo. 1881. Gli scamiciati. Seguito alla Milano sconosciuta. Milano: Ambrosoli.

Verga, Giovanni. 2003. "Lultima giornata". In Per le vie, edizione critica a cura di Raffaele Morabito, 108-114. Firenze: Le Monnier.

Verga, Giovanni. 2008. “Tentazione!”. In Per le vie e altre novelle lombarde, a cura di Vincenzo Guarracino, 193-200. Milano: Otto/Novecento. 